\title{
Formulation, Stability and Physicochemical Properties of Pickering Emulsions: An Overview
}

\author{
Papa Mady $\mathrm{Sy}^{1}$, Sidy Mouhamed Dieng ${ }^{1} \&$ Mounibé Diarra ${ }^{1}$ \\ ${ }^{1}$ Laboratory of Physics and Pharmaceutical Biophysics, Faculty of Medicine, Pharmacy and Odontology, UCAD, \\ Dakar, Senegal \\ Correspondence: Papa Mady Sy, Laboratory of Physics and Pharmaceutical Biophysics, Faculty of Medicine, \\ Pharmacy and Odontology, UCAD, Dakar, Senegal. E-mail: madyspa@gmail.com; mounbeni@yahoo.fr
}

Received: October 23, 2018

Accepted: November 22, $2018 \quad$ Online Published: January 29, 2019

doi:10.5539/apr.v11n1p41

URL: http://dx.doi.org/10.5539/apr.v11n1p41

\begin{abstract}
This review work focuses mainly on the formulation, characterization, physicochemical properties and parameters of stability of emulsions stabilized by solid particles (Pickering emulsions). This concept of emulsions stabilized by particles strong knows a renewed interest in our days saw the benefits they present: good stability, protection of the environment, safety of users, types of particles etc. The adsorption of the solid particles at the oil-water interface is almost irreversible and strong, leading to the formation of a dense film, creating a barrier around the droplets and thus making the droplets very resistant to coalescence. Recently, the possibilities of application of emulsions stabilized by particles are considered in the pharmaceutical industry. This type of formulation may be a potential system of encapsulation of the active ingredients, allowing controlled and targeted release of the active ingredient from the internal phase.
\end{abstract}

Keywords: Pickering-emulsions-stability-Physico-chemical properties

\section{Introduction}

Pickering emulsions are dispersions of two thermodynamically unstable, immiscible liquids stabilized by solid particles. This fine-particle stabilization effect, known for about a century, was first demonstrated by Ramsden (Ramsden, 1904) and then described more fully by Pickering (Pickering, 1907). The latter has established that particles, like surfactants, can stabilize oil-in-water emulsions. Such emulsions have not yet given rise to major industrial developments, but they are experiencing a renewed interest in the socio-economic context, especially since their stability is often better than that of emulsions stabilized by surfactants. classics (Fouilloux, 2011). It has been shown that the emulsions thus obtained can, like those stabilized by molecular surfactants, be of direct $(\mathrm{O} / \mathrm{W})$, inverse $(\mathrm{W} / \mathrm{O})$ or multiple $(\mathrm{W} / \mathrm{O} / \mathrm{W}$ or $\mathrm{O} / \mathrm{W} / \mathrm{O})$ type. (Aveyard et al., 2003; Binks, 2002). This stabilization of Pickering emulsions is related to the adsorption of particles at the interface between the immiscible phases (Marku et al., 2012).

\section{Pickering Emulsion Formulation, Characterization and Stabilization Concepts}

\subsection{Formulation and Characterization of Pickering Emulsions}

\subsubsection{Formulation}

A drop can be divided into several droplets of smaller size under the action of mechanical forces: pressure, velocity gradient, or turbulent forces. But overall, fractionation results from the competitive effects between the rheological and interfacial properties of the two phases, and the hydrodynamic characteristics of the flow via mechanical forces (Theron, 2009). Before breaking a drop, the interface separating the continuous phase from the dispersed phase deforms under the action of tangential and normal forces. The surface tension $\gamma$ allows a spherical drop of radius $\mathrm{R}$ to withstand a stress of the same intensity as the pressure difference on either side of the interface, called Laplace's law (Saad \& Neumann, 2016):

$$
\Delta \mathrm{P}=\frac{2 \gamma}{\mathrm{R}}
$$

There are a variety of techniques for forming emulsions. The most used are: 
$\checkmark$ Rotor stator system: The principle is based on the fractionation of a drop into several droplets of smaller size under the action of a mechanical force;

$\checkmark$ Ultrasonication: The principle is based on the irradiation of a liquid medium with sound waves below $20 \mathrm{kHz}$.

Two mechanisms make it possible to explain the effect of ultrasound on emulsification:

$\checkmark$ The wave generates instabilities at the liquid-liquid interface, which will induce the formation of droplets;

$\checkmark$ The implosion of cavitation bubbles near the drops of liquid will break these drops and there is a gradual reduction of the average diameter of the emulsion (Retamal Marín et al., 2017).

These techniques have significant advantages: ease of implementation, ability to quickly prepare large volumes of emulsion. However, the resulting emulsions are generally polydispersed, making systematic studies and the rationalization of such systems difficult (Abismail et al., 1999). They also make it possible to create large amounts of interface, in order to disperse one phase in another to form an emulsion. It is then possible to disperse an apolar phase in a polar phase, leading to an oil-in-water emulsion or on the contrary to disperse a polar phase in an apolar phase leading to a water-in-oil emulsion (Fouilloux, 2011). Several types of particles can be used for the stabilization of Pickering emulsions: iron oxide, metal hydroxides, silica, clays, carbon (Aveyard et al., 2003; Wang \& Hobbie, 2003). Stabilizing particles are not necessarily solid. Effective stabilization has been observed with microgel particles, liquid crystals, but also with natural particles such as bacteria and spores (Binks \& Whitby, 2005; Dorobantu et al., 2004; Fujii et al., 2005).

\subsubsection{Characterization}

\subsubsection{Determining the Direction of the Emulsion}

The nature of the external phase, and therefore the type of emulsion, can be detected by various techniques:

$\checkmark$ By dispersibility experiments of a small volume of emulsion in an aqueous or oily phase: For example, if the sample taken is dispersible in water (respectively in oil) the emulsion is of type $\mathrm{H} / \mathrm{E}$ (respectively $\mathrm{E} / \mathrm{H}$ ) (Zoller, 2008);

$\checkmark$ By conductivity measurements: In most cases, water, unlike oil, contains electrolytes. The conductivity of the aqueous phase is therefore 100 to 1000 times higher than that of the oil. Since the value of the conductivity of an emulsion depends on its external phase, it is therefore relatively easy to determine whether the emulsion is of W/O or $\mathrm{O} / \mathrm{W}$ type. The conductivity of an emulsion $\left(\chi_{e m}\right)$ is directly related to the conductivity of the external phase $\left(\chi_{e x t}\right)$ and its volume fraction $\left(f_{w}\right)$. Thus, as a first approximation, the following linear relationship can be used:

$$
\chi_{e m}=f_{w} \cdot \chi_{\text {ext }}
$$

The external phase can also be of paramount importance in the interpretation of phenomena observed for emulsions, in particular for detecting phase inversion during an emulsification process (Pizzino et al., 2009);

$\checkmark$ By dye tests: The principle of these techniques is based on the determination of the water solubility or liposolubility of dyes such as methylene blue or Nile red (Sudan III) in the emulsion formed.

\subsubsection{Determination of Droplets Size}

One of the important properties of emulsions is the size of the droplets, which can be determined by a measurement method called "particle size". The size of the droplets is particularly representative of the stirring and formulation conditions in which the emulsion has been prepared. In most cases, an emulsion contains droplets of different sizes, in particular because of the partly or totally random character of the agitation processes, and therefore a droplet size distribution which represents a statistical inventory of the population present in the emulsion. The emulsion can be characterized by a single diameter value representative of an average of the total drop population (Querol et al., 2017).

\subsubsection{Determination of Viscosity}

The rheological behaviour of an emulsion is often complex because of the influence of many parameters inherent in the structure (size and organization of the droplets) or the chemical compounds used. Many models that exist in the literature relate the viscosity of an emulsion to its characteristics, however they are mostly of empirical nature and limited to specific cases. The viscosity of an emulsion depends on the viscosity of the external phase, which is generally expressed by the following relationship:

$$
\eta_{\text {em }}=\eta_{\text {ext }} f(\text { other variables })
$$


where "em" et "ext" refer respectively to the emulsion and the external phase. $f$ represents the contribution of the effects of the other variables (droplet sizes etc. ...) and tends to unity when the volume of the droplets tends to zero. This equation is often written in the form:

$$
\eta_{r}=\frac{\eta_{e m}}{\eta_{e x t}}
$$

$\eta_{r}$ is the relative viscosity. The rheological behavior of an emulsion often depends on the viscosity of its external phase (Theron, 2009).

\subsubsection{Determination of $\mathrm{pH}$}

$\mathrm{pH}$ plays a very important role in the stability of emulsions. The contact angle, the adsorption of the particles at the interface, the Zeta potential and other factors are parameters influenced by the $\mathrm{pH}$. The resistance of the particle dispersion structure is enhanced by the increase in $\mathrm{pH}$, and their adsorption at the oil/water interface is controlled by $\mathrm{pH}$. The zeta potential decreases with increasing $\mathrm{pH}$. The contact angle also increases with increasing $\mathrm{pH}$ but the variation is small. In sum, the stability of emulsions increases with increasing $\mathrm{pH}$ (Yang et al., 2007).

\subsection{Energy Aspects and Interactions between Particles at the Interface}

\subsubsection{Energy Aspects}

The mere presence of solid particles is not sufficient for the formation of emulsions. The latter are formed by energy input, corresponding to the energy required to create a larger interfacial area (Colver, 2008; Frelichowska et al., 2010). The energy of the attachment of a particle to the interface of a fluid is related not only to the angle, but also to the interfacial tension. This energy therefore determines the stability of the emulsion and increases as the contact angle approaches $90^{\circ}$ (Powell \& Chauhan, 2014). Colloidal particles are often irreversibly adsorbed at the water/oil interface. Assuming that a spherical solid particle is small (submicronic) so that the effect of gravity is negligible, the energy E needed to move a particle of radius $r$ from the oil/water interface to one of the volume phases is given by the following equation:

$$
\Delta \mathrm{E}=\gamma_{\mathrm{HE}} \pi r^{2}(1 \pm \cos \theta)^{2}
$$

$\Delta \mathrm{E}=$ Energie variation

$\mathrm{r}=$ droplet radius

$\gamma_{\mathrm{HE}}=$ surface tension between oil-water

$\theta=$ contact angle

The positive sign in the equation corresponds to the extraction towards the oily phase and the negative sign to the extraction towards the aqueous phase (Binks, 2002; Pichot et al., 2010; Prestidge \& Simovic, 2006; RondónGonzález et al., 2007). This equation shows that depending on the contact angle, the adsorption of a particle to the surface can be high or low (10kBT). It is weak for contact angles between 0 and $20^{\circ}$ or $160^{\circ}$ and $180^{\circ}$ and maximum for angles close to $90^{\circ}$. For the contact angle close to $90^{\circ}$, the energy required to remove a particle from the interface is of the order of a few thousand kBT (Popp et al., 2010; Tarimala, 2006). A consequence of the very high attachment energy of the particles at the interface with respect to the kBT thermal energy is that the particles, once at the interface, can be as irreversibly adsorbed at the interface (Pickering, 1907).

\subsubsection{Interactions between Particles at the Interface}

Van der Waals interactions bring together all molecular interactions of dipolar origin. We distinguish the so-called Keesom interactions, which occur between permanent dipoles, and the Debye interactions that appear between the induced dipole of a non-polar molecule and a permanent dipole. A third contribution, called London, corresponds to induced dipole/induced dipole interaction. It is always present and due to the fact that a spontaneous dipole moment, related to the fluctuations of position of the electronic cloud around the nuclei can appear at any moment. This dipole then induces the formation of another dipole within a neighboring molecule, and the interaction between these dipoles is at the origin of attractive forces (Figure 8) (Etienne \& David, 2012). For the interaction between two spherical particles, the sum of these contributions is written as:

$$
\mathrm{U}(\mathrm{d})=-\mathrm{A} \frac{\mathrm{r}}{12(\mathrm{~d}-2 \mathrm{r})}
$$

U: interaction Energy,

d: the distance separating the particles, 
r: particles radius,

A: the Hamaker constant which depends on the molecular properties of the material.

The Van der Waals interactions between two particles are generally of effective range of a few tens of nanometers and exhibit a divergent evolution in contact between the particles. The Hamaker constant is always positive for two bodies of the same chemical nature, resulting in an attractive interaction, but it can be negative for bodies of different chemical nature, which generates a repulsive interaction (Wen et al., 2004).

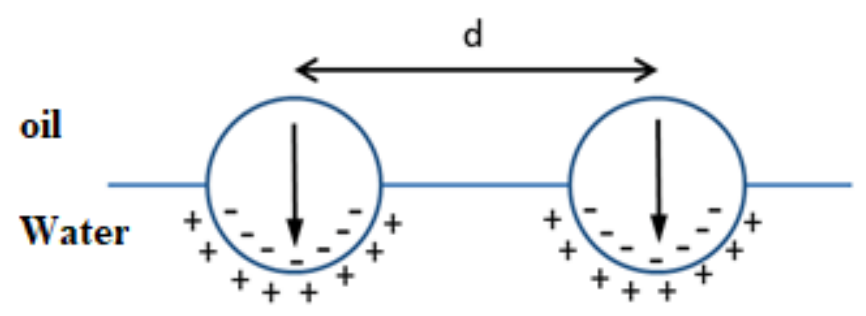

Figure 1. Diagram of the cloud of counter-ions of particles located at a water/oil interface and dipole moment generated (Ridel, 2015)

\section{Type, Stability and Instability of Pickering Emulsions}

\subsection{Experimental Factors Influencing the Type of Emulsion}

Several experimental factors influence the position of the particles at the interface, and consequently the stability of the emulsions.

\subsubsection{Particles Wettability}

The physicochemical surface properties of the particles determine their wettability by liquids. Indeed, Schulman and Leja (Schulman \& Leja, 1954) published the first study on a variation of the wettability of barium sulphate particles and the influence of the contact angle on the stabilization of emulsions. They observed that the type of emulsion obtained depends directly on the value of the contact angle between solid, oily and aqueous phase.

Surface chemical treatment can alter the wettability of the particles. For example, in the case of silica particles, the grafting of hydrocarbon chains on the surface renders them hydrophobic. Depending on the degree of grafting, it is possible to obtain a whole range of hydrophilic, partially hydrophobic or totally hydrophobic silicas, which can be expressed by the amount of residual free hydroxyl groups on the surface. Such particles, while not amphiphilic, have affinity for water/oil interfaces (Binks \& Lumsdon, 2000b).

Moreover the modification of the surface of the particles can take place in situ. The oxides or metals are chemically modified by the adsorption of short-chain carboxylic acids or amines on their surface. It is also possible to transform certain hydrophobic particles into hydrophilic particles, or vice versa, by $\mathrm{pH}$ change (Akartuna et al., 2008; Gonzenbach et al., 2007; Wang et al., 2005). Binks and Lumsdon (Binks \& Lumsdon, 2000a) have shown that the same silica particles can stabilize water-in-oil emulsions at $\mathrm{pH}<9$ and $\mathrm{O} / \mathrm{W}$ emulsions at $\mathrm{pH}>12.5$. This phase inversion is due to the change in the distribution of the silica particles between the aqueous and oily phase. At high $\mathrm{pH}$, the silanol $\mathrm{SiOH}$ groups dissociate to $\mathrm{SiO}_{2}$, which slightly increases the wetting of the particles with water and promotes the formation of oil-in-water emulsions. In the case of a mixture of hydrophobic and hydrophilic particles stabilizing a fixed oil/water ratio emulsion, increasing the fraction of one of the types of particles can lead to a transitional reversal of the phases. For example, in a water-in-oil emulsion stabilized by hydrophobic silica, the addition of the hydrophilic silica leads to the production of an oil-in-water emulsion (Binks \& Lumsdon, 2000a). This phenomenon is similar to that observed in the case of emulsions stabilized by surfactants where the addition of a hydrophobic co-emulsifier in the presence of a hydrophilic emulsifier causes the emulsion to be inverted (Fouilloux, 2011).

\subsubsection{Initial Location of Particles}

One possible explanation for the influence of the initial particle location is the difference in hydrophobicity for wetted particles by water and oil. For particles that adsorb at the interface entering the water side, the effective contact angle is the recoil contact angle (in water). On the contrary, it is the advancing angle (in water) when particles are adsorbed from the oil. Since the angles at the recoil are smaller than the advancing angles, the same particles behave as if they were more hydrophilic, so with a preference for the stabilization of the $\mathrm{O} / \mathrm{W}$ emulsions. The opposite situation is observed for particles with a forward angle: they are more hydrophobic, so with a 
preference for $\mathrm{W} / \mathrm{O}$ emulsions. Optimal stability can therefore be achieved by simply changing the initial location of the particles (Binks \& Rodrigues, 2003; Fouilloux, 2011). However, the value of the contact angle alone does not determine the type of the emulsion obtained, in particular, the particles having a contact angle close to $90^{\circ}$ can stabilize the two types of emulsion. The interactions between the two phases of the emulsion and the stabilizing particles play an important role during the introduction of the second phase of the emulsion. Yan et al. (Yan \& Masliyah, 1993) demonstrated that particles must reside in the outer emulsion phase before emulsification to stabilize emulsions. If the hydrophobic particles are dispersed in water prior to emulsification, they are unlikely to pass the interface barrier and enter the oily phase. Significant energy input would be required to force these particles to cross the interface (Asekomhe et al., 2005).

\subsection{Experimental Factors Influencing Stability}

\subsubsection{Size and Shape of Particles}

The size of the particles determines their potential to remain in suspension and their adsorption at the interface. Experimentally, it has been shown that a decrease in particle size, at least to a certain extent, increases the stability of the emulsions because the size of droplets formed to a minimum size decreases (Tambe \& Sharma, 1994). To date, there is no systematic study in the literature on the influence of the polydispersity of nanoparticles on the stabilization of Pickering emulsions. The shape and roughness of the surface of the particles have an influence on their wettability. These two values also impact the hysteresis of the value of the contact angle. To simplify calculations and analysis, Pickering's emulsion stabilization models generally consider spherical particles. However, in practice, the stabilizing particles are often anisotropic or rough. Thus, the clay particles have a disk shape, and the fumed silica particles widely used for the Pickering emulsion formulation form stable threedimensional aggregates composed of spherical particles (Asekomhe et al., 2005; Binks \& Lumsdon, 1999; Yan et al., 2001). There is currently no systematic study of the role of particle shape in the stabilization of Pickering emulsions. This is probably one of the least well-controlled factors.

\subsubsection{State of Dispersion of the Particles}

The dispersing power of the particles can be defined as their properties of being able to keep solid particles suspended in a liquid (Begbeg, 2008). Aggregated particles adsorb more strongly at the interface. Studies have shown that a slight flocculation of the particles by addition of salt or $\mathrm{pH}$ variation leads to stable emulsions but high flocculation leads to negative results (Colver, 2008). For oil/water type emulsions this flocculation is obtained by addition of electrolytes. The addition of electrolytes such as $\mathrm{NaCl}$ during the formulation causes a decrease in the zeta potential with increasing $\mathrm{pH}$ leading to the aggregation of LDH particles into larger flakes. The strength of the LDH dispersions structure is enhanced by the increase in $\mathrm{pH}$ and particle concentration. A slight variation in the contact angle has also been observed (Yang et al., 2007). It is reported that flocculated dispersions are more effective in stabilizing emulsions because particles that are not flocculated have more mobility at the interface than flocculated particles that easily coalesce (Jin-Hwang et al., 2004; Pickering, 1907).

\subsubsection{Particles Concentration}

The droplet size of the emulsion decreases as the amount of particles increases relative to the constant amount of dispersed phase. This occurs up to a minimum droplet size and the excess particle appears in the outer phase (Binks \& Whitby, 2004; Tambe \& Sharma, 1994). The high concentration of particles does not mean a dense overlap of the interface. The Yan and Levine teams (Levine \& Sanford, 1985; Yan et al., 2001) have observed that particles are in the outer emulsion phase, even when the surface of the droplets is not fully covered. But for a concentration too small in particles, the average size of the droplets increases over time. Addition of the particles in the outer phase after emulsion preparation causes gelation of the outer phase and does not cause size reduction (Arditty et al., 2003; Binks \& Lumsdon, 2000a). The formation of a three-dimensional network of flocculated particles in the outer phase around the droplets has been observed when the quantity of particles is sufficient. This improves the stability by hindering the mutual contact of the droplets. For example, clay particles form a network in water and surround oil droplets (Abend \& Lagaly, 2001; Thieme et al., 1999).

\subsection{Phenomena of Instability}

An emulsion is thermodynamically unstable. The stability of the emulsions can only be achieved if the formulator is able to slow down or inhibit the physical mechanisms that normally lead to the demixing of the immiscible phases (Brochette, 2013). These mechanisms are explained below.

\subsubsection{Sedimentation and Creaming}

Sedimentation and creaming are the result of the same phenomenon, whose engine is gravity. Because the system is necessarily in kinetic equilibrium, a dispersed phase droplet is animated by a Brownian motion induced by the 
solvent molecules, the momentum being preserved during solvent/particle shocks. Thus the same droplet is subjected to the gravitational field, which tends to impose a downward movement if the droplet is denser than the continuous phase or upward otherwise (Brochette, 2013). This competition between Brownian agitation and gravity results in an inhomogeneity of the emulsion left without agitation.

Consider the case of an emulsion water in oil left at rest; the concentration in droplets may decrease with height in the manner of a decreasing exponential. If the volume $\mathrm{v}$ of the droplets is large, the majority of the droplets will be collected at the bottom of the storage tube, and we can then observe a sedimentation pellet (Brochette, 2013). If an oil-in-water $\mathrm{O} / \mathrm{W}$ emulsion had been considered, the distribution law would be identical, but there would be an accumulation of droplets in the upper part of the storage tube: this is known as creaming (Abend \& Lagaly, 2001; Begbeg, 2008; BROCHETTE, 2013; Langevin et al., 2004; Robins, 2000; Thieme et al., 1999; Torres et al., 2008). Sedimentation or creaming of the emulsified globules results in a loss of homogeneity evident to the naked eye. The speed of the process is given by Stokes' law (Briant, 1989; Hamoudi, 2012; Langevin et al., 2004):

$$
\mathrm{v}=\frac{2 \mathrm{r}^{2} \mathrm{~g}\left(\mathrm{D}_{1}-\mathrm{D}_{2}\right.}{9 \mathrm{n}}
$$

$\mathrm{v}=$ Vitesse of sedimentation $(\mathrm{m} / \mathrm{s})$

$\mathrm{r}=$ droplets radius $(\mu \mathrm{m})$

$\mathrm{g}=$ acceleration due to earth gravity $\left(\mathrm{m} / \mathrm{s}^{2}\right)\left(\mathrm{g}=9.807 \mathrm{~m} / \mathrm{s}^{2}\right)$

$\mathrm{D}_{1} ; \mathrm{D}_{2}=$ respective densities of the dispersed phase and the continuous phase

$\eta=$ viscosity of the continuous phase $\left(\mathrm{cP}_{\mathrm{o}}\right)$

In order to avoid these phenomena we can act on the speed $v$ by (Briant, 1989):

$\checkmark$ increase in the viscosity of the continuous phase by addition of thickeners that may cause a change in the nature of the interfacial barriers,

$\checkmark$ decrease in the droplet diameter by acting on the stirring speed,

$\checkmark$ decrease in the density difference between the two phases,

$\checkmark$ avoid aggregation of the droplets.

\subsubsection{Floculation}

Sometimes the droplets formed do not remain independent of each other but tend to cluster to form clusters. This phenomenon, called flocculation, is often a precursor to the sedimentation of the clusters thus formed (Briant, 1989). The flocculation originates from a droplet adhesiveness, the origin of which is a competition between thermal agitation and Van der Waals forces (Brochette, 2013). The droplets of an emulsion are in fact animated by a Brownian motion, which induces shocks between droplets. If a sufficient attractive interaction exists between the droplets thus brought into contact, they remain associated. In order to understand, then control the flocculation of emulsions, it is necessary to know the basic theory on which it is based. Emulsions being liquid dispersions, it can be considered that DLVO theory of hydrophilic colloidal emulsions is applicable to them.

Taking into account the influence of interfacial layers likely to modify this theory, this approach can be used semiquantitatively, but no theory can predict the stability of long-term emulsions. There are many reasons for this (Briant, 1989):

$\checkmark$ the heterogeneity of the particle size distribution and the variations of this distribution with coalescence;

$\checkmark$ the mobility of the emulsions both by lateral passage at the point of contact of two particles and by diffusion through the interfacial layer to the aqueous phase or to the oily phase;

$\checkmark$ changes in the emulsion due to ripening;

$\checkmark$ the need to have a mechanical barrier.

$\checkmark$ To avoid this phenomenon, it is necessary to (Galenica., 1983):

$>$ avoid creaming and sedimentation (because these phenomena put the droplets in contact),

$>$ increase steric and electrostatic repulsions (using ionic surfactants, for example).

\subsubsection{Coalescence}

Flocculation, sedimentation and creaming are reversible phenomena (weak agitation allows "redispersion"). This is not the case with coalescence, which is the phenomenon of ultimate degradation of emulsions (BROCHETTE, 
2013). This irreversible mechanism follows the reconciliation of two or more globules, then the thinning followed by the rupture of the thin continuous phase film located between the droplets (Begbeg, 2008; Cheng \& Velankar, 2009; Langevin et al., 2004; Rondon Anton, 2006; Tcholakova et al., 2008).

To avoid this phenomenon, it is necessary to (Galenica., 1983):

$>$ prevent flocculation,

$>$ enhance the strength of the film by the choice of surfactant or solid particles.

\subsubsection{Ostwald Ripening}

Ostwald ripening depends partly on granulometry and secondly on the Laplace overpressure (BRIANT, 1989; BROCHETTE, 2013). At the end of the emulsification step, the population of droplets is not homogeneous in size. Generally, a particle size approaching a log-normal distribution is observed (Rojas \& Patricia, 2007). There is a flow of matter from small droplets to large droplets, through the continuous phase. The small droplets are emptied in favor of the large ones, and the granulometry is modified since the classes of small sizes disappear. This irreversible phenomenon is the ripening of Ostwald (Galenica, 1983; Langevin et al., 2004; Thieme et al., 1999; Xu et al., 2005).

This phenomenon can be avoided by (Begbeg, 2008; Galenica., 1983):

$\checkmark$ monodispersion of the droplet population,

$\checkmark$ decreased solubility by adding salt in a W/O emulsion or a high molar mass apolar solute for an $\mathrm{O} / \mathrm{W}$ emulsion, $\checkmark$ surfactants barrier to the diffusion of molecules of the dispersed phase.

\subsubsection{Phase Inversion}

For some authors, phase inversion is a phenomenon of degradation of the emulsion. This analysis is correct if we consider that during the inversion, the continuous phase of the emulsion becomes the dispersed phase and vice versa, and that the properties of the diphasic medium are disrupted. On the other hand recent studies that are the fruit of half a century of research, suggests that the phenomena to be taken into account are different from those described by previous studies.

Through inversion, it is possible to obtain emulsions with particular characteristics (for example, a very small size of the droplets) under conditions completely different from those described by the research on direct emulsification (Rondón-González, 2007).

Phase inversion can be produced either by:

$\checkmark$ the change of a formulation variable, such as temperature, salinity (ionic surfactants) or surfactant,

$\checkmark$ the change in a composition variable, such as the fraction of the aqueous phase or the concentration of surfactant or alcohol used.

The first type of inversion is defined as transitional, while the second is called catastrophic (Rondón-González et al., 2007).

\section{Applications of Pickering Emulsions}

Although the phenomenon of the stabilization of emulsions by particles has been known for a long time, their applications are still under development. Knowledge of Pickering's emulsion stabilization mechanisms is necessary in many branches of industry, when the formulation of these emulsions is expected or when it is a technological problem. The use of Pickering emulsions is a new strategy for the encapsulation of active ingredients for controlled release in the pharmaceutical and cosmetic formulation. Two properties of Pickering emulsions appear to be particularly interesting, the very good stability and the specific interfacial properties. The assembly of nanoparticles with fluid interfaces, as is the case with the stabilization of Pickering emulsions, is a method of forming a shell around droplets (Radulova et al., 2018). This type of structure corresponds to encapsulation systems which have the advantages of: encapsulating and protecting labile active principles against degradation by an unfavorable pH medium or by enzymes of the digestive fluids (Marefati et al., 2017; Shao et al., 2018; Sy et al., 2018), modulating the important fundamental biological process of lipid digestion by virtue of their distinctive stability against coalescence and resilience to desorption by intestinal biosurfactants (Sarkar et al., 2018). Pickering emulsions can also be used in drug delivery systems (Sy et al., 2018; Sy et al., 2018; Wang et al., 2017).

In the cosmetic field, the main interest of Pickering emulsions for a dermopharmaceutical use is the absence of emulsifiers which are often responsible for irritation phenomena, foaming, haemolysis, strong interactions with biological membranes. The presence of particles in the formulation can reinforce the protection against UV rays. 
Indeed, studies have been conducted to obtain emulsions stabilized exclusively with titanium dioxide particles to obtain emulsions without surfactant and with good sun protection (Marto et al., 2016).

\section{Conclusion}

Pickering emulsions are surfactant free emulsions stabilized by solid particles. These systems are gaining renewed interest on the one hand, because it is preferable to limit the use of synthetic surfactants for ecological reasons, and on the other hand, because the functionalization of particles has seen recent progress. Pickering emulsions, for example, are systems used in the pharmaceutical industry to protect active ingredients, serve as reservoirs and allow controlled release. They can increase the solubility and the therapeutic index of certain active principles. In cosmetology, Pickering's emulsions have great potential for cosmetic formulation and many patents are currently appearing on this application.

\section{References}

Abend, S., \& Lagaly, G. (2001). Bentonite and double hydroxides as emulsifying agents. Clay Minerals, 36, 557570.

Abismaïl, B., Canselier, J. P., Wilhelm, A. M., Delmas, H., \& Gourdon, C. (1999). Emulsification by ultrasound: Drop size distribution and stability. Ultrasonics Sonochemistry, 6, 75-83.

Akartuna, I., Studart, A. R., Tervoort, E., Gonzenbach, U. T., \& Gauckler, L. J. (2008). Stabilization of Oil-inWater Emulsions by Colloidal Particles Modified with Short Amphiphiles. Langmuir, 24, 7161-7168.

Arditty, S., Whitby, C. P., Binks, B. P., Schmitt, V., \& Leal-Calderon, F. (2003). Some general features of limited coalescence in solid-stabilized emulsions. Eur. Phys. J. E, 11, 273-281.

Asekomhe, S. O., Chiang, R., Masliyah, J. H., \& Elliott, J. A. W. (2005). Some Observations on the Contraction Behavior of a Water-in-Oil Drop with Attached Solids. Ind. Eng. Chem. Res., 44, 1241-1249.

Aveyard, R., Binks, B. P., \& Clint, J. H. (2003). Emulsions stabilised solely by colloidal particles. Advances in Colloid and Interface Science, 100-102, 503-546.

Begbeg, A. (2008). Importance des considérations environnementales dans l'étude des performances des additifs utilises dans les fluides de forage (Thesis).

Binks, B. P. (2002). Particles as surfactants Tosimilarities and differences. Interface Science, 21.

Binks, B. P., \& Lumsdon, S. O. (1999). Stability of oil-in-water emulsions stabilised by silica particles. Physical Chemistry Chemical Physics, 1, 3007-3016.

Binks, B. P., \& Lumsdon, S. O. (2000a). Effects of oil type and aqueous phase composition on oil-water mixtures containing particles of intermediate hydrophobicity. Phys. Chem. Chem. Phys., 2, 2959-2967.

Binks, B. P., \& Lumsdon, S. O. (2000b). Influence of Particle Wettability on the Type and Stability of SurfactantFree Emulsions. Langmuir, 16, 8622-8631.

Binks, B. P., \& Rodrigues, J. A. (2003). Types of Phase Inversion of Silica Particle Stabilized Emulsions Containing Triglyceride Oil. Langmuir, 19, 4905-4912.

Binks, B. P., \& Whitby, C. P. (2004). Silica Particle-Stabilized Emulsions of Silicone Oil and Water: Aspects of Emulsification. Langmuir, 20, 1130-1137.

Binks, B. P., \& Whitby, C. P. (2005). Nanoparticle silica-stabilised oil-in-water emulsions: Improving emulsion stability. Colloids and Surfaces A: Physicochemical and Engineering Aspects, 253, 105-115.

BRIANT, J. (1989). Phénomènes d'interface. Agents de surface: Principes et modes d'action. Editions TECHNIP.

BROCHETTE, P. (2013). Émulsification-Élaboration et étude des émulsions [WWW Document]. Ref: TIP453WEB-“Formulation.” Retrieved September 26, 2018 from https://www.techniques-ingenieur.fr/basedocumentaire/procedes-chimie-bio-agro-th2/cosmetiques-procedes-de-formulation42634210/emulsification-j2150/

Cheng, H. L., \&Velankar, S. S. (2009). Controlled Jamming of Particle-Laden Interfaces Using a Spinning Drop Tensiometer. Langmuir, 25, 4412-4420.

Colver, P. J. (2008). Using Pickering stabilisation as a tool for the fabrication of supra-colloidal structures (Ph.D.). University of Warwick.

Dorobantu, L. S., Yeung, A. K. C., Foght, J. M., \& Gray, M. R. (2004). Stabilization of Oil-Water Emulsions by Hydrophobic Bacteria. Appl. Environ. Microbiol., 70, 6333-6336. 
Etienne, S., \& David, L. (2012). Introduction à la physique des polymères-2e éd. Dunod.

Fouilloux, S. (2011). Nanoparticules et microfluidique pour un système modèle d'émulsions de Pickering. Etude des mécanismes de stabilisation et déstabilisation (Theses). Université Pierre et Marie Curie-Paris VI.

Frelichowska, J., Bolzinger, M. A., \& Chevalier, Y. (2010). Effects of solid particle content on properties of O/W Pickering emulsions. Journal of Colloid and Interface Science, 351, 348-356.

Fujii, S., Read, E. S., Binks, B. P., \& Armes, S. P. (2005). Stimulus-Responsive Emulsifiers Based on Nanocomposite Microgel Particles. Advanced Materials, 17, 1014-1018.

Galenica.: Agents de Surface Et Emulsions. Les systemes disperses, 1. (1983). Technique et Documentation.

Gonzenbach, U. T., Studart, A. R., Tervoort, E., \& Gauckler, L. J. (2007). Tailoring the Microstructure of ParticleStabilized Wet Foams. Langmuir, 23, 1025-1032.

Hamoudi, M. C. (2012). Beads made of cyclodextrin and oil for oral delivery of lipophilic drugs (Theses). Université Paris Sud-Paris XI.

Jin-Hwang, K., Song, I. K., Kee-Yeol, K., Eun-Joo, L., \& Moung-Seok, Y. (2004). Effect of Aqueous Phase Composition on the Stability of a Silica-stabilized Water-in-oil Emulsion. Journal of the Society of Cosmetic Scientists of Korea, 30, 353-359.

Langevin, D., Poteau, S., Hénaut, I., \& Argillier, J. F. (2004). Crude Oil Emulsion Properties and Their Application to Heavy Oil Transportation. Oil \& Gas Science and Technology, 59, 511-521.

Levine, S., \& Sanford, E. (1985). Stabilisation of emulsion droplets by fine powders. The Canadian Journal of Chemical Engineering, 63, 258-268.

Marefati, A., Bertrand, M., Sjöö, M., Dejmek, P., \& Rayner, M. (2017). Storage and digestion stability of encapsulated curcumin in emulsions based on starch granule Pickering stabilization. Food Hydrocolloids, 63, 309-320.

Marku, D., Wahlgren, M., Rayner, M., Sjöö, M., \& Timgren, A. (2012). Characterization of starch Pickering emulsions for potential applications in topical formulations. International Journal of Pharmaceutics, 428, 17.

Marto, J., Gouveia, L. F., Gonçalves, L., Chiari-Andréo, B. G., ..., \& Ribeiro, H. M. (2016). Design of novel starch-based Pickering emulsions as platforms for skin photoprotection. Journal of Photochemistry and Photobiology B: Biology, 162, 56-64.

Pichot, R., Spyropoulos, F., \& Norton, I. T. (2010). O/W emulsions stabilised by both low molecular weight surfactants and colloidal particles: The effect of surfactant type and concentration. Journal of Colloid and Interface Science, 352, 128-135.

Pickering, S. U. (1907). CXCVI.-Emulsions. J. Chem. Soc., 91, 2001-2021.

Pizzino, A., Catté, M., Van Hecke, E., Salager, J. L., \& Aubry, J. M. (2009). On-line light backscattering tracking of the transitional phase inversion of emulsions. Colloids and Surfaces A: Physicochemical and Engineering Aspects, 338, 148-154.

Popp, N., Kutuzov, S., \& Böker, A. (2010). Various Aspects of the Interfacial Self-Assembly of Nanoparticles. In A. H. E. Müller \& H. W. Schmidt (Eds.), Complex Macromolecular Systems II, Advances in Polymer Science (pp. 39-58). Springer Berlin Heidelberg, Berlin, Heidelberg.

Powell, K. C., \& Chauhan, A. (2014). Interfacial Tension and Surface Elasticity of Carbon Black (CB) Covered Oil-Water Interface [WWW Document].

Prestidge, C. A., \& Simovic, S. (2006). Nanoparticle encapsulation of emulsion droplets. International Journal of Pharmaceutics, Selected papers from the 15th International Microencapsulation Symposium, 324, 92-100.

Querol, N., Barreneche, C., \& Cabeza, L. F. (2017). Method for controlling mean droplet size in the manufacture of phase inversion bituminous emulsions. Colloids and Surfaces A: Physicochemical and Engineering Aspects, 527, 49-54.

Radulova, G. M., Slavova, T. G., Kralchevsky, P. A., Basheva, E. S., Marinova, K. G., \& Danov, K. D. (2018). Encapsulation of oils and fragrances by core-in-shell structures from silica particles, polymers and surfactants: The brick-and-mortar concept. Colloids and Surfaces A: Physicochemical and Engineering Aspects, 559, 351-364. 
Ramsden, W. (1904). Separation of solids in the surface-layers of solutions and 'suspensions' (observations on surface-membranes, bubbles, emulsions, and mechanical coagulation). Preliminary account. Proc. R. Soc. Lond., 72, 156-164.

Retamal Marín, R. R., Babick, F., \& Stintz, M. (2017). Ultrasonic dispersion of nanostructured materials with probe sonication-practical aspects of sample preparation. Powder Technology, 318, 451-458.

Ridel, L. (2015). Pickering emulsions: Theoretical approach and applications: Interfacial physico-chemical analysis: Obtention of Pickering nanoemulsions by spontaneous emulsification and Pickering aerated emulsions (Theses). Université Claude Bernard-Lyon I.

Robins, M. M. (2000). Emulsions-creaming phenomena. Current Opinion in Colloid \& Interface Science, 5, 265272.

Rojas, R., \& Patricia, M. (2007). Emulsification en cuve agitée: Rôle du protocole opératoire sur l'inversion de phase catastrophique (Thesis). Toulouse, INPT.

Rondon Anton, M. (2006). Influence de la formulation physico-chimique et des propriétés interfaciales sur la stabilité des émulsions asphaltènes-eau-huile: Application à la deshydratation du pétrole (Thesis). Pau.

Rondón-González, M. (2007). Inversion de phase d'émulsions induite par agitation (Thesis). Vandoeuvre-lesNancy, INPL.

Rondón-González, M., Madariaga, L. F., Sadtler, V., Choplin, L., Márquez, L., \& Salager, J. L. (2007). Emulsion Catastrophic Inversion from Abnormal to Normal Morphology 6. Effect of the Phase Viscosity on the Inversion Produced by Continuous Stirring. Industrial \& Engineering Chemistry Research, 46, 3595-3601.

Saad, S. M. I., \& Neumann, A. W. (2016). Axisymmetric Drop Shape Analysis (ADSA): An Outline. Advances in Colloid and Interface Science, 238, 62-87.

Sarkar, A., Zhang, S., Holmes, M., \& Ettelaie, R. (2018). Colloidal aspects of digestion of Pickering emulsions: Experiments and theoretical models of lipid digestion kinetics. Advances in Colloid and Interface Science.

Schulman, J. H., \& Leja, J. (1954). Control of contact angles at the oil-water-solid interfaces. Emulsions stabilized by solid particles (BaSO4). Trans. Faraday Soc., 50, 598-605.

Shao, P., Zhang, H., Niu, B., \& Jin, W. (2018). Physical stabilities of taro starch nanoparticles stabilized Pickering emulsions and the potential application of encapsulated tea polyphenols. International Journal of Biological Macromolecules, 118, 2032-2039.

Sy, P. M., Anton, N., Idoux-Gillet, Y., Dieng, S. M., ..., \& Vandamme, T. F. (2018). Pickering nano-emulsion as a nanocarrier for $\mathrm{pH}$-triggered drug release. International Journal of Pharmaceutics, 549, 299-305.

Sy, P. M., Djiboune, A. R., Diouf, L. A. D., Soumboundou, M., ..., \& Diarra, M. (2018). Water/Oil Pickering Emulsion Stabilized by Magnesium Oxide Particles: A Potential System with Two Active Substances (Paracetamol and Griseofulvin). Open Journal of Biophysics, 8, 68.

Tambe, D. E., \& Sharma, M. M. (1994). The effect of colloidal particles on fluid-fluid interfacial properties and emulsion stability. Advances in Colloid and Interface Science, 52, 1-63.

Tarimala, S. (2006). Self-assembled structure and dynamics of solid particles at Pickering emulsion interfaces.

Tcholakova, S., Denkov, N. D., \& Lips, A. (2008). Comparison of solid particles, globular proteins and surfactants as emulsifiers. Phys. Chem. Chem. Phys., 10, 1608-1627.

Theron, F. (2009). Conception et mise en ouvre d'un procédé intensifié continu de microencapsulation par polycondensation interfaciale ( $\mathrm{PHd})$.

Thieme, J., Abend, S., \& Lagaly, G. (1999). Aggregation in Pickering emulsions. Colloid. Polym. Sci., 277, 257260.

Torres, L., Iturbe, R., Snowden, M. J., Chowdhry, B., \& Leharne, S. (2008). Can Pickering emulsion formation aid the removal of creosote DNAPL from porous media? Chemosphere, 71, 123-132.

Wang, D., Duan, H., \& Möhwald, H. (2005). The water /oil interface: The emerging horizon for self-assembly of nanoparticles. Soft Matter, 1, 412-416.

Wang, H., \& Hobbie, E. K. (2003). Amphiphobic Carbon Nanotubes as Macroemulsion Surfactants. Langmuir, 19, 3091-3093. 
Wang, J., Law, W. C., Chen, L., Chen, D., \& Tang, C. Y. (2017). Fabrication of monodisperse drug-loaded poly(lactic-co-glycolic acid)-chitosan core-shell nanocomposites via pickering emulsion. Composites Part B: Engineering, Bio-inspired Nano-engineered Materials, 121, 99-107.

Wen, L., Cheng, J., Zou, H., Zhang, L., Chen, J., \& Papadopoulos, K. D. (2004). van der Waals Interaction between Internal Aqueous Droplets and the External Aqueous Phase in Double Emulsions. Langmuir, 20, 8391-8397.

Xu, Q. Y., Nakajima, M., \& Binks, B. P. (2005). Preparation of particle-stabilized oil-in-water emulsions with the microchannel emulsification method. Colloids and Surfaces A: Physicochemical and Engineering Aspects, 262, 94-100.

Yan, N., Gray, M. R., \& Masliyah, J. H. (2001). On water-in-oil emulsions stabilized by fine solids. Colloids and Surfaces A: Physicochemical and Engineering Aspects, 193, 97-107.

Yan, Y., \& Masliyah, J. H. (1993). Solids-stabilized oil-in-water emulsions: Scavenging of emulsion droplets by fresh oil addition. Colloids and Surfaces A: Physicochemical and Engineering Aspects, 75, 123-132.

Yang, F., Niu, Q., Lan, Q., \& Sun, D. (2007). Effect of dispersion pH on the formation and stability of Pickering emulsions stabilized by layered double hydroxides particles. Journal of Colloid and Interface Science, 306, 285-295.

Zoller, U. (2008). Handbook of Detergents-6 Volume Set. CRC Press.

\section{Copyrights}

Copyright for this article is retained by the author(s), with first publication rights granted to the journal.

This is an open-access article distributed under the terms and conditions of the Creative Commons Attribution license (http://creativecommons.org/licenses/by/4.0/). 